\title{
Peer Review of "Peer review of "Burst Beliefs - Methodological Problems in the Balloon Analogue Risk Task and Implications for Its Use""
}

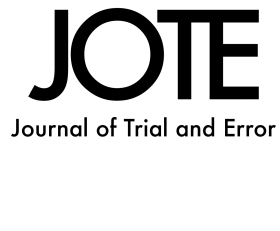

Michael Young ${ }^{1}$, Sihua $\mathrm{Xu}^{2}$

\author{
${ }^{1}$ Reviewer 1 \\ ${ }^{2}$ Reviewer 2 \\ This is the originally submitted version of \\ "Peer review of "Burst Beliefs - \\ Methodological Problems in the Balloon \\ Analogue Risk Task and Implications for Its \\ Use"” with peer review comments attached. \\ Original author(s): \\ Kristel De Groot. \\ Published version: \\ https://doi.org/10.36850/mr1. \\ How to read: \\ Sections which have peer review comments \\ are marked by $\nabla$ or links, clicking which \\ directs you to said comments. The reviews \\ contain links referring back to the original \\ section.
}

\begin{abstract}
Studies in the field of psychology often employ (computerised) behavioural tasks, aimed at mimicking real-world situations that elicit certain actions in participants. Such tasks are for example used to study risk propensity, a trait-like tendency towards taking or avoiding risk. One of the most popular tasks for gauging risk propensity is the Balloon Analogue Risk Task (BART; Lejuez et al., 2002), which has been shown to relate well to self-reported risk-taking and to real-world risk behaviours. However, despite its popularity and qualities, the BART has several methodological shortcomings, most of which have been reported before, but none of which are widely known. In the present paper, four such problems are explained and elaborated on: a lack of clarity as to whether decisions are characterised by uncertainty or risk; censoring of observations; confounding of risk and expected value; and poor decomposability into adaptive and maladaptive risk behaviour. Furthermore, for every problem, a range of possible solutions is discussed, which overall can be divided into three categories: using a different, more informative outcome index than the standard average pump score; modifying one or more task elements; or using a different task, either an alternative risk-taking task (sequential or otherwise), or a custom-made instrument. It is important to make use of these solutions, as applying the BART without accounting for its shortcomings may lead to interpretational problems, including false positive and false negative results. Depending on the research aims of a given study, certain shortcomings are more pressing than others, indicating the (type of) solutions most needed. By combining solutions and openly discussing shortcomings, researchers may be able to modify the BART in such a way that it can operationalise risk propensity without substantial methodological problems.

KEYWORDS

Balloon Analogue Risk Task, risk-taking, uncertainty, expected value, confounding, censoring.
\end{abstract}

\section{Purpose}

The Balloon Analogue Risk Task (BART) is one of the most widely used behavioural tasks in psychology, and has an especially strong presence in the fields of decision research, addiction research, and neuropsychology. But despite its popularity, researchers using the BART seem largely unaware of the task's methodological shortcomings, sometimes leading to conclusions that are not supported by the data. This is likely a result of these shortcomings not being widely reported, as 'failure' is not considered a popular publishing theme. Therefore, the present paper aims to collect and review these shortcomings, as well as potential solutions.

\section{Take-home Message}

The Balloon Analogue Risk Task (BART) suffers from various methodological shortcomings. The present paper analyses these shortcomings, and offers suggestions to mitigate their effects. Finally, it calls upon researchers to critically evaluate how these shortcomings impact their studies before deciding whether and how to use BART.

\section{Introduction}

To a large extent, psychological science rests on the promises of operationalisation: defining fuzzy concepts as measurable variables, or in other words, 
changing conceptual variables into operational ones (Shuttleworth, 2008). This process is imperative as most concepts researchers hypothesise about are not straightforwardly quantifiable. By defining how a concept is measured, operationalisation allows hypotheses to take a falsifiable format and enables us to replicate findings. In a way, operationalisations are arbitrary, as concepts can be defined and thus measured in numerous ways - none of which are surely 'right'. Nonetheless, some measures may be more suitable than others.

A notable example of a concept that can be operationalised in various ways is risk-taking, which has an important place in clinical, cognitive, and developmental psychology, as well as in the fields of criminology, economics, and management (Lauriola \& Weller, 2018). One way in which risk-taking is operationalised in these fields is through self-report measures, such as the Domain-Specific Risk-Taking (DOSPERT) scale (Blais \& Weber, 2006) and the Financial Risk Tolerance assessment (Grable \& Lytton, 1999). Another way is through computerised behavioural tasks, like the Iowa Gambling Task (Bechara, Damasio, Damasio, \& Anderson, 1994), the Cambridge Gambling Task (Rogers et al., 1999), the Game of Dice Task (Brand et al., 2005), the Balloon Analogue Risk Task (Lejuez et al., 2002), and the more recent but already widely used Columbia Card Task (Figner, Mackinglay, Wilkening, $\&$ Weber, 2009). Importantly, the quality of a study largely depends on the degree to which its operational measures reflect the underlying concept; in this case, one's disposition towards taking risk. If a task is a poor proxy for a concept or is subject to methodological or interpretational problems, any data resulting from it are of limited value to our understanding of the concept. In this regard, several studies have challenged the operationalisation ability of the most-cited risk task, the Iowa Gambling Task (see e.g. Brand, Labudda, \& Markowitsch, 2006; Buelow \& Suhr, 2009; Figner et al., 2009; Maia \& McClelland, 2004). The Balloon Analogue Risk Task, which is the secondmost cited, may yet suffer from even severer issues, hindering its ability to operationalise risk-taking. While some individual issues have been reported in previous publications, no literature so far has discussed these collectively. The present commentary aspires to fill this gap.

\section{The Balloon Analogue Risk Task}

In the Balloon Analogue Risk Task, or BART for short, participants are presented with a computer screen showing a small balloon and a pump. They are told that every time they click the pump, the balloon expands, and a fixed amount of money ( 5 cents) is added to a temporary bank. Every pump also increases the chance of the balloon exploding (marked by a 'pop' sound from the computer), resulting in losing all money in the temporary bank for that particular balloon (trial). The point at which a balloon explodes varies across trials, ranging from the first pump to the point where the balloon fills the entire screen. Participants can decide to stop pumping the balloon at any point during a trial by clicking the 'collect' button (left in Figure 1), which transfers the money accumulated in their temporary bank to their permanent one, while a slot machine sound is played. Once a balloon explodes or once participants cash a balloon's proceeds, the trial ends, and a new, uninflated, balloon appears.

In the original study by Lejuez et al. (2002), participants were informed that they would complete 90 balloons: 30 orange, 30 yellow, and 30 blue ones. Unbeknownst to participants, differently coloured balloons had a different chance of exploding. The probability distribution governing their explosion points consisted of an array of $n$ numbers from which on every pump a random number was drawn without replacement. If a 1 was drawn, the balloon exploded. Thus, the probability $p$ of the balloon exploding on the first pump was $1 / n$, and the probability of it exploding on pump $i$ (given no prior explosion) was

$$
p_{i}=\frac{1}{n-i+1}
$$

For orange balloons, the array ranged from 1 to 8 (hence $p_{1}=\frac{1}{8-1+1}=1 / 8$ ), for yellow balloons from 1 to $32\left(p_{1}=\frac{1}{32-1+1}=1 / 32\right)$, and for blue ones from 1 to $128\left(p_{1}=\frac{1}{128-1+1}=1 / 128\right)$. Their average explosion points were respectively 4,16 , and 64 , with the same (randomly generated) sets of explosion points being used across all participants to limit extraneous variability. Neither the ranges nor the average explosion points were communicated to participants.

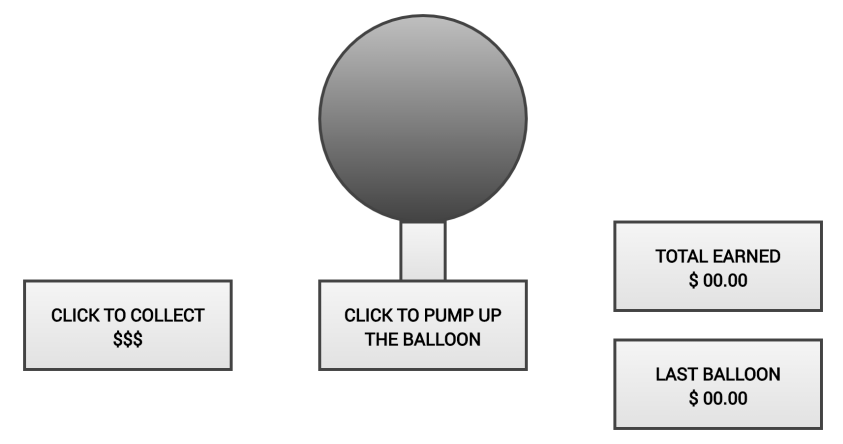

FIG URE 1 Set-up of the original Balloon Analogue Risk Task as described by Lejeuz (2002). An interactive illustration of the task is provided with the HTML version of this article.

The BART's design is intended to reflect naturalistic decision-making, in which taking more risk generally increases the odds of encountering a loss. This sort of decision-making tends to be emotionally engaging, instigating a sense of increasing tension as the balloon grows bigger (Schonberg, Fox, \& Poldrack, 2011). In support of the BART's validity, the average number of times participants pump the blue balloon significantly correlates with scores on risk-related constructs (sensation seeking, impulsivity) and with real-world risk behaviours, such as polydrug use, gambling, unsafe sex, and stealing (Lejuez et al., 2002). The orange and yellow pumps were originally not examined in relation to risk-related constructs, as their narrow ranges of outcome values (1-8 and 1-32) are less suited for capturing individual differences. Instead, their average pump numbers were analysed together with those of the blue balloons to show that the number of times participants choose to pump is sensitive to the probability of exploding. Overall, the data showed the BART to have "particular promise as a behavioural index of risk-taking" (Lejuez et al., 2002, p. 82). As would be expected based on this conclusion, the BART (particularly its blue balloon version) became a popular instrument for gauging individuals' propensity for taking risk, with inconsistent findings being attributed to factors like sampling variability and inadequate statistical power (Lauriola, Panno, Levin, \& Lejuez, 2014), rather than problems inherent to the BART. However, several authors have argued that such problems exist (e.g. De Groot \& Thurik, 2018; Gu, Zhang, Luo, Wang, \& Broster, 2018; Schmidt, Kessler, Holroyd, \& 
Miltner, 2019; Schonberg et al., 2011), and that they limit the BART's ability to measure one's propensity to take risk. The key problems in the task are 1) a lack of clarity as to whether decisions are characterised by uncertainty or risk, 2) censoring of observations, 3) confounding of risk and expected value, and 4) poor decomposability into adaptive and maladaptive risk behaviour.

\section{| Risk or Uncertainty?}

In economic theories of decision-making, a key distinction is that between uncertainty and risk, which is often accredited to Knight (1921), and was introduced to psychological thinking in a seminal paper by Edwards (1954) that lies at the origin of behavioural decision theory. When making a decision under the condition of risk, the probabilities associated with the possible outcomes are known. When deciding under uncertainty (which some authors call ambiguity), this probability distribution is unknown.

For Knight (1921), this distinction was not only of theoretical, but of practical importance as well. According to him, uncertainty - not risk - was the main driver of entrepreneurial success, as only people who recognise hidden opportunities can seize them and profit from them. Since then, the empirical relevance of the uncertainty-risk distinction has been confirmed in various fields of research. In economics, Ellsberg (1961) showed that individuals prefer risk over uncertainty, even if the known probabilities are unfavourable and the uncertain option could be a guaranteed win. In psychology, studies showed that uncertain and risky decisions involve different mental processes, as risk allows for statistical thinking (to optimise) but uncertainty involves heuristics (to satisfice) (Volz \& Gigerenzer, 2012). In line with this, decision-making under risk is thought to depend more on executive function (such as categorisation and cognitive flexibility), for which the dorsolateral prefrontal cortex is important, whereas decision-making under uncertainty hinges on emotional processes (such as somatic feedback), which are more associated with the ventromedial prefrontal cortex and the amygdala (Brand et al., 2006). This may explain why patients with executive deficits, such as those with Parkinson's disease, have difficulty deciding under risk but have no trouble deciding under uncertainty (Euteneuer et al., 2009), whereas persons with obsessive-compulsive disorder, for example, show the opposite pattern (Starcke, Tuschen-Caffier, Markowitsch, \& Brand, 2009, 2010).

Given that uncertainty and risk differ both theoretically and empirically, it is imperative for researchers to know the conditions under which participants decide. Unfortunately, despite the word 'risk' in its name, these conditions are not straightforward in the BART. Since participants are never given "detailed information about the probability of an explosion" (Lujuez et al., 2002, p. 77), we can assume that at least during early trials, they decide under uncertainty (Bishara et al., 2009; De Groot \& Thurik, 2018; Schonberg et al., 2011). As they move further along in the task and 'sample the distribution' by pumping balloons and observing their outcomes, they learn more about the probabilities, and gradually shift towards deciding under risk. Such a shift has also been observed in the Iowa Gambling Task, in which performance in early trials does not correlate with that in later trials nor with executive function, indicating that people first decide under uncertainty and later under risk (Brand et al., 2006; Brand, Recknor, Grabenhorst, \& Bechara, 2007).

The transition in the BART from deciding under uncertainty to deciding under risk is problematic for a number of reasons. First, it is unclear when exactly this shift transpires, making it difficult to determine whether a decision in a given trial is made under uncertainty, risk, or something in between. Second, the point where decisions shift from uncertainty to risk is likely to differ between individuals, and is dependent on task characteristics (Brand et al., 2006; Brand et al., 2007). Third, the shift implies that the BART imposes learning demands, which could inadvertently impact participants' outcomes on the task, with those capable of updating their knowledge of the probabilities performing better than those who have difficulty doing so. Fourth, once participants manage to derive the task's probabilities, subsequent decisions are not characterised by what is usually considered risk. Contrary to decisions in which probabilities are explicitly described ('a priori' probabilities), probabilities in the BART are derived from experience. Since such probabilities depend on factors like sampling variability and one's memory of previous events, decision-makers treat experience-based probability differently, which is called the description-experience gap (Hau, Pleskac, Kiefer, \& Hertwig, 2008; Rakow \& Newell, 2010). Most notably, when deciding based on experience, people do not act in accordance with prospect theory, but instead underweight rare events and overweight common encounters. As people have more and more encounters (e.g. trials), their experiences will approach the precision of a priori probabilities, though in practice this is difficult to attain (Knight, 1921).

To address the inability of the BART to differentiate between complete uncertainty, experience-based risk, and description-based risk, several approaches may be used. One option is to apply a model to the BART's data that allows for participants learning through experience. An early example is a model by Wallsten, Pleskac, and Lejuez (2005) in which decision-makers update their probabilities from trial to trial, and continually re-evaluate their options. Alternatively, one could use a different task, in which decisions are either all characterised by uncertainty or risk, or which includes a well-understood shift between the two. For instance, some tasks involve only decisions made under (a priori) risk, like the Cambridge Gambling Task, the Game of Dice Task, and the Columbia Card Task, the latter of which resembles the BART's dynamic, affective nature (Schonberg et al., 2011). Unfortunately, no task with a well-understood shift has been reported yet, although the shift in the Iowa Gambling Task has been studied more thoroughly than that in the BART.

\section{| Censored Observations}

Statistical censoring refers to a condition in which the value of an observation is unknown because it is beyond a certain limit. This limit can exist by design, which is common in survival analysis. If a study on a surgical intervention follows patients for up to 10 years, the longevity scores of those who live past this term are censored, as their longevity is at least 10 (Young \& McCoy, 2019). Censoring can also result from limits on what an instrument can reliably measure. For example, the full IQ score of the Wechsler Adult Intelligence Scale ranges from 40 to 160 (Sattler \& Ryan, 2009), meaning that IQ scores of people performing either extremely poorly or extremely well are cut off at these boundaries and are thus censored.

In the BART, censoring (by design) occurs if a participant is stopped from taking more risk in a given trial, because the balloon they are pumping explodes, forcing the trial to end. Since such a trial ends prematurely, the number of 
times the participant pumped the balloon does not necessarily reflect the risk they were willing to take, meaning their risk propensity is censored. This is problematic for various reasons. First, including these censored trials biases the average number of pumps downwards (especially for high-risk takers), underestimating participants' willingness to take risks (Dijkstra, Tiemeier, Figner, \& Groenen, 2020; Pleskac, Wallsten, Wang, \& Lejuez, 2008). Likewise, the between-subjects variability across these averages is reduced (Lejuez et al., 2002). Overall, the (unadjusted) average number of pumps is an ill-suited operationalisation of risk propensity.

As censoring affects all sequential risk-taking tasks like the BART (involving multiple decisions per trial) and various other research paradigms, like survival analysis, several solutions have been proposed. In the paper introducing the BART, Lejuez et al. (2002) suggest computing an adjusted pump average using only trials in which participants stopped voluntarily, that is, in which the balloon did not burst. However, by omitting explosion trials, censored observations are essentially treated as randomly missing, which is inaccurate (Pleskac et al., 2008). The more risk someone takes, the more likely it is that the balloon bursts, and that the trial forcedly ends. The termination of trials is therefore not independent from participants' behaviour. As a result, Lejuez et al.'s adjusted score tends to discard trials in which participants take a lot of risk. This causes the average number of pumps to be biased downwards, similar to the unadjusted score, but to a lesser extent.

To circumvent the problem of censoring, Pleskac et al. (2008) developed an automatic response version of the BART. Contrary to the standard BART, in which participants inflate a balloon one pump at a time, the automatic BART lets them indicate their intended number of pumps beforehand. The balloon then inflates to the corresponding size, or until it bursts. This procedure allows for an unbiased statistic of risk propensity, as the intended number of pumps is now observable in all trials (Pleskac et al., 2008). However, it increases the time between decision and outcome, which may make decisions less emotional (impulsive) and more cognitive (planned) (Pleskac et al., 2008), and may reduce the salience of the outcomes (Young \& McCoy, 2019). These effects, in turn, can reduce participants' risk-taking.

Another solution to censoring is using a rigged task (Slovic, 1966). Participants are then told that failure can occur at any moment (in the BART, at any pump), but actually, it is set to occur at the last possible choice. Hence, participants can always stop voluntarily, and no scores are censored. To uphold credibility, 'mock' trials are added, in which failure is set to occur early on. Deciding on the number and timing of mock trials, however, is a challenge. Since behaviour in a trial is affected by previous outcomes, experiencing (too) few failures could increase risk-taking (De Groot \& Van Strien, 2019; Dijkstra et al., 2020). Therefore, rigged tasks should be designed such that they produce failure rates similar to non-rigged tasks, and should take into account that failure rates differ between participants too. However, research on the Columbia Card Task, another sequential risk-taking task, shows that this is often not the case (De Groot \& Van Strien, 2019).

A final remedy, which addresses the bias but leaves the BART unchanged, is to apply a statistical model to the resulting data that explicitly incorporates censored behaviour. Such models consider all observed data, using the censored trials as lower bounds in determining a participant's actual risk propensity.
Some of them employ Bayesian (generalised) linear mixed-effects regression (Weller, King, Figner, \& Denburg, 2019; Young \& McCoy, 2019); others use maximum likelihood estimation, adding a cumulative distribution function to the likelihood function to account for censoring (Dijkstra et al., 2020; Tobin, 1958). Such models perform significantly better (i.e., have less biased predictions) than those that do not account for censoring. However, as is the case for all statistical models, their soundness hinges on the validity of their underlying assumptions (Schafer \& Graham, 2002), such as that of normality, whose violation not all models are robust against (Powell, 1984).

\section{| Confounding and Decomposability}

The BART was designed to resemble real-world risk situations, where taking modest risk is generally advantageous, but taking excessive risk is increasingly unfavourable (Lejuez et al., 2002; Wallsten et al., 2005). Within a trial, every successful pump earns participants 5 cents, which are added to their temporary bank. As the amount accumulated in the bank grows, the relative gain of taking additional risk decreases, while the potential loss in case of an explosion increases. Additionally, the probability of the balloon exploding increases with every pump: from 1/128 on the first, to $1 / 127$ on the second, and so on.

This combination of characteristics makes that the task's structure entails a serious problem. While the balloon value increases linearly, the probability of the balloon exploding increases superlinearly, so that the expected value of pumping the balloon - the product of the success chance and the reward, minus the product of the explosion chance and the amount participants already have in their temporary bank - changes across a trial (Schmidt et al., 2019). This change is illustrated in Table 1. Early in a trial, the expected value of the pump is positive, so taking additional risk is advantageous. This prospect changes halfway, when the expected value turns negative, making additional pumps unfavourable (Lejuez et al., 2002). Due to the expected value changing with each decision, it is confounded with risk (defined as the variability of the possible outcomes), which varies across decisions by design. This makes it difficult to measure participants' risk propensity, as both risk and expected value may influence their decisions. The extent to which individuals are, for example, risk-seeking, can therefore not be determined, as this would require showing a preference for higher variance payoffs, holding expected value constant (Schonberg et al., 2011).

This confounding demonstrates that the BART's main observable outcome - the number of pumps participants press - cannot be interpreted as a straightforward indicator of risk propensity. Like many behavioural tasks, the BART supposedly gauges a single cognitive construct, but actually manipulates various other, potentially confounding constructs as well (Schonberg et al., 2011). Expected value is an example of such a construct. As a result, the single score provided by the BART cannot easily be decomposed to identify the cognitive or neural mechanisms involved in the pump decisions. Studying the risk-taking process in isolation using the BART is therefore not possible.

One approach for resolving the confounding and decomposability issues in the BART is to apply a computational model to its data that quantifies the cognitive mechanisms underlying the observed behaviour (Bishara et al., 2009). Such models were first proposed by Wallsten et al. (2005), inspired by an 
expectancy-valence model for decomposing behaviour in the Iowa Gambling Task (Busemeyer \& Stout, 2002). Wallsten et al. explain decision variability using a parameter for risk-taking, one for response consistency, and two for learning. By applying these models, we can study risk-taking - and other aspects that determine BART behaviour - in isolation, by translating "what is observed but relatively uninformative to what is unobserved and relatively informative" (Van Ravenzwaaij, Dutilh, \& Wagenmakers, 2011, p. 95). However, data from the BART may not be rich enough to warrant the use of complicated decomposition models. For instance, a study on Wallsten et al.'s best performing model demonstrated that its learning parameters could not reliably be recovered (Van Ravenzwaaij et al., 2011). To allow for more extensive decomposition, one may need to resort to a different task, like the Iowa Gambling Task. Alternatively, one could use a task that by design avoids confounding, such as the Columbia Card Task. Although dynamic and affective like the BART, this task orthogonally varies risk-related constructs, so that they can be decomposed into their underlying mechanisms - like sensitivity to gains, losses, and probabilities - without the use of a computational model (Dijkstra et al., 2020; Figner et al., 2009; Schonberg et al., 2011). Finally, researchers can choose to design a custom task to ensure that the constructs relevant to their hypotheses are not confounded. For example, a risk task presented in Schmidt, Mussel, and Hewig (2013) varies the level of risk, but holds expected value constant. Solutions such as these should be considered carefully, so that constructs crucial to a study's hypotheses can be isolated effectively.

\section{| The Normative Solution}

The BART is designed in such a way that the balloons' average explosion point lies at 64, halfway the maximum number of pumps. This is achieved by randomly generating collections of explosion points until one produces an average of 64 over all trials, as well as within each set of 10 trials (Lejuez et al., 2002). Participants can then maximise their earnings by attempting to pump every balloon 64 times, which results in an explosion in about half of the trials, and an optimal overall expected value. Going back to Table 1, we can see exactly why this is the optimal, or normative, solution in the BART. Up to and including the $64^{\text {th }}$ pump, the expected value of pumping the balloon is positive; after 64 , the expected value is (increasingly) negative. It is therefore optimal to aim for 64 pumps on every balloon, and then stop. Choosing to pump more or fewer than 64 times will decrease expected earnings; and the farther one deviates from the optimum, the lower the expected earnings become (Lejuez et al., 2002; Pleskac et al., 2008; Wallsten et al., 2005).

Remarkably, in the majority of trials, participants stop pumping the balloon far before the optimal stopping point (Lejuez et al., 2002). In fact, the average adjusted pump score is typically between 26 and 35 (Pleskac et al., 2008). Real-world risk-avoiders and risk-takers alike rarely pump the balloon enough times to maximise their expected earnings. This is less of a problem in the automatic BART, although participants there still pump fewer than 64 times on average. For example, two recent studies reported averages of 61.9 (Bernoster, De Groot, Wieser, Thurik, \& Franken, 2019) and 58.5 pumps (De Groot \& Van Strien, 2019).

It is yet unknown exactly why participants often stop pumping before they reach the optimal point, but various factors may play a role. First, since the original BART requires participants to inflate balloons one pump at a time, it is plausible that they get tired of pumping before reaching the optimum. Second, people might become satiated: due to diminishing marginal returns, adding 5 cents to a growing temporary bank might stop being an attractive prospect well before reaching pump 64 . Third, participants may need time to learn which strategy results in maximal earnings (Lejuez et al., 2002). This conjecture is supported by the observation that participants in both the original and the automatic BART on average press more pumps in the final block of 10 trials than they do in previous blocks (Lejuez et al., 2002; Author \& Author, year). ${ }^{1}$ It also corresponds with the presumed shift from deciding under uncertainty to deciding under risk. However, learning the optimal solution is hard, as the range of possible explosion points is large (0-128), and individual explosions provide limited feedback. This is in line with finding by Lejuez et al. (2002), who show that larger explosion ranges result in larger relative deviations from the optimum.

The fact that participants in the BART often stop pumping before the optimal stopping point has serious implications for how the data can be interpreted. Up to 64 pumps, the risk they take can be characterised as adaptive or functional, as it results in higher earnings. After that point, it can be considered maladaptive or dysfunctional, as it reduces expected earnings. Since people generally pump fewer than 64 times, the BART cannot properly differentiate between adaptive and maladaptive risk behaviour, neither within nor between participants. A second, related problem is that experimental manipulations meant to increase risk-taking (such as adding time pressure or administering a certain drug) generally do not lead to lower earnings, as even the resulting higher pump numbers usually do not exceed 64 (Pleskac et al., 2008). For example, if a manipulation causes participants to take more risk and press 50 instead of 30 times, they are actually, on average, better off than before, the opposite of what one would expect in real life. In short, if participants mostly stay under 64 pumps, they simply never reach the point where taking more risk becomes disadvantageous, which limits the conclusions one can draw from the data.

The most straightforward way to mitigate these problems may be the modified BART developed by Pleskac et al. (2008), which differs from the original task in three ways. First, it involves an automatic response mode: participants indicate their intended number of pumps at the start of each trial, after which the balloon automatically inflates to the corresponding size (or until it bursts). Although meant to mitigate censoring, this adjustment may also prevent people from getting tired of pumping. Second, the adjusted task provides explicit feedback about the explosion point of every balloon, not merely of those that actually explode. This may improve participants' learning across trials. Third, participants are (truthfully) informed that the range of pump numbers is 1-128, and that the best overall number of pumps is 64 , further increasing the amount of information at their disposal.

These three modifications together successfully moved participants' behaviour closer to the normative solution of 64 , with an average pump score of 57.7 for females and 63.7 for males (Pleskac et al., 2008). Part of this effect can be attributed to the automatic response mode, as these averages are higher than those from a manual BART with full feedback and strategy instructions

1. The relevant data collected by Author and Author (year) on per-block averages is not reported in the published report, but will be shared upon request. 


\begin{tabular}{|c|c|c|c|c|c|c|}
\hline $\begin{array}{l}\text { Pump Number } \\
\text { (A) }\end{array}$ & $\begin{array}{l}\text { Balloon Value } \\
\text { Before Pump } \\
\text { (B) }\end{array}$ & $\begin{array}{l}\text { Balloon Value } \\
\text { After Pump } \\
\text { (C) }\end{array}$ & $\begin{array}{l}\text { Chance of } \\
\text { Explosion } \\
\text { (D) }\end{array}$ & $\begin{array}{l}\text { Chance of } \\
\text { Success } \\
\text { (E) }\end{array}$ & $\begin{array}{l}\text { Expected Value of } \\
\text { Current Pump } \\
\text { (F) }\end{array}$ & $\begin{array}{l}\text { Expected Value of } \\
\text { All Remaining } \\
\text { Pumps (G) }\end{array}$ \\
\hline 2 & $€ 0.05$ & $€ 0.10$ & 0.00787 & 0.99213 & $€ 0.04921$ & $€ 1.56260$ \\
\hline 3 & $€ 0.10$ & $€ 0.15$ & 0.00794 & 0.99206 & $€ 0.04840$ & $€ 1.52540$ \\
\hline 5 & $€ 0.20$ & $€ 0.25$ & 0.00806 & 0.99194 & $€ 0.04798$ & $€ 1.45161$ \\
\hline \multicolumn{7}{|l|}{$(\ldots)$} \\
\hline 62 & $€ 3.05$ & $€ 3.10$ & 0.01493 & 0.98507 & $€ 0.00373$ & $€ 0.00672$ \\
\hline 63 & $€ 3.10$ & $€ 3.15$ & 0.01515 & 0.98485 & $€ 0.00227$ & $€ 0.00303$ \\
\hline 66 & $€ 3.25$ & $€ 3.30$ & 0.01587 & 0.98413 & $€-0.00238$ & $€-0.00238$ \\
\hline \multicolumn{7}{|l|}{$(\ldots)$} \\
\hline 124 & $€ 6.15$ & $€ 6.20$ & 0.20000 & 0.80000 & $€-1.19000$ & $€-1.19000$ \\
\hline 125 & $€ 6.20$ & $€ 6.25$ & 0.25000 & 0.75000 & $€-1.51250$ & $€-1.51250$ \\
\hline 126 & $€ 6.25$ & $€ 6.30$ & 0.33333 & 0.66667 & $€-2.05000$ & $€-2.05000$ \\
\hline 127 & $€ 6.30$ & $€ 6.35$ & 0.50000 & 0.50000 & $€-3.12500$ & $€-3.12500$ \\
\hline 128 & $€ 6.35$ & $€ 6.40$ & 1.000000 & 0.00000 & $€-6.35000$ & $€-6.35000$ \\
\hline
\end{tabular}

TAB LE 1 Changing Balloon Values, Explosion and Success Chances, and Expected Values Across Balloon Pumps. Note: The expected value of the current pump (F) is computed by multiplying the success chance $(E)$ by 0.05 , then subtracting the product of the explosion chance (D) and the balloon value before the pump (B) $[F=E * 0.05-D * B]$. Alternatively, one can also take into account the expected value of any subsequent pumps, insofar as they are advantageous $(G)$. This results in somewhat different values, but an identical tipping point at 64 .

added. Since this manual BART itself resulted in higher averages than the original BART, the feedback and instructions likely also contributed to the effect (Lejuez et al., 2002). Recent research, however, indicates that informing participants about the optimal strategy is not necessary, and even ill-advised. Two studies using an automatic BART with full feedback - but without strategy instructions - found equally high pump averages as did Pleskac and colleagues (Bernoster et al., 2019; De Groot \& Van Strien, 2019). In addition, these studies showed that a subgroup of participants - often from a STEM background seem to infer the optimal strategy without any help. Their repeated 64-answers therefore reflect cognitive ability rather than risk propensity, and reduce task variability. Informing participants about the optimal strategy can increase such problematic responses. Therefore, it seems best to add automatic responses and full feedback to the BART, but not strategy instructions. This will likely elicit sufficiently high pump averages, without compromising the validity of the task.

\section{Discussion}

Since it was first published in 2002, the BART has become one of the most popular tools in psychology to gauge individuals' propensity for taking risk. Halfway 2020, the original article describing the BART (Lejuez et al., 2002) had been cited over 1100 times in Scopus, most often in journals on decision research, addiction, and neuropsychology. This popularity is well-founded. The BART succeeds in recreating the 'natural' feeling of exhilaration and tension people experience when taking risk, and thus has excellent ecological validity. Furthermore, it correlates well with self-reported risk-related constructs, such as impulsivity and sensation-seeking, and with real-world risk behaviours, like polydrug use and unsafe sex, supporting its convergent validity. Lastly, it does not correlate with constructs like depression and anxiety, endorsing its discriminant validity (Lejuez et al., 2002). But despite these qualities, the BART suffers from methodological problems, most of which have been acknowledged in previous research as negatively impacting its rigour. The present paper is the first to give a comprehensive overview of these problems.

The first problem concerns the lack of clarity as to whether decisions in the BART are made under uncertainty (where outcome probabilities are unknown) or risk (where they are known). Since participants are not given any information about the explosion probabilities, they first decide under uncertainty, which then gradually shifts towards risk as they learn more about the probabilities in the task. As it is unclear exactly when this shift takes place, it is difficult to determine whether a given decision is made under uncertainty, risk, or something in between. The second problem concerns statistical cen- 
soring, which occurs in trials where the balloon explodes, as participants are then prevented from taking additional risk. As a result, the average number of times participants pump the balloon underestimates their risk propensity. Third, the BART confounds risk with expected value. Since these constructs change simultaneously throughout a trial, participants' pump behaviour again does not reflect risk propensity, as decisions are influenced by both risk and expected value. This also means that the task is poorly decomposable, as it cannot disentangle the motives underlying a pump decision. A final problem concerns the task's normative solution. In the majority of trials, participants stop pumping before the point where expected earnings are maximised. Therefore, participants mostly take adaptive risk, which leads to higher earnings. Maladaptive risk-taking hardly occurs, even though one would expect to see such behaviour in certain cases.

Despite these problems, much of the research up to now has focused on the empirical findings produced by the BART, rather than on the task itself, with the majority of researchers using the task without critically reviewing whether its problems interfere with their aims. This can have undesirable consequences, such as when it leads to false positives or false negatives. For example, one may fail to show a relationship which only exists for decisions characterised by risk, as some trials in the BART are characterised by uncertainty instead. Conversely, a hypothesis may pertain to people's response to changing risk and be unjustly supported, as in the BART, risk and expected value simultaneously change and impact individuals' behaviour. Finding true positives and negatives hinges on several factors, an important one being the validity of the measurement instrument. Any data resulting from instruments that suffer from methodological or interpretational problems is of limited value to understanding the concepts they are supposed to operationalise.

For these reasons, it is imperative that researchers critically evaluate the 'fit' between their research and the BART before deciding on using it. For many research aims, one will now see that the original BART does not suffice. Yet despite these 'burst beliefs', there are three types of approaches one can take to account for its limitations. First, data from the original BART can be analysed using a different, more informative index than Lejuez et al.'s average adjusted pump score. For example, the models by Wallsten et al. (2005) break down behaviour into risk-taking, response consistency, and learning. In addition, computational models can be used to take into account censoring and to provide an index of uncensored risk-taking in the BART (Dijkstra et al., 2020; Tobin, 1958; Weller et al., 2019; Young \& McCoy, 2019). A second way of dealing with the BART's limitations is by modifying the task, for example by rigging it (Figner et al., 2009; Slovic, 1966), providing additional feedback, or automating the responses (Pleskac et al., 2008). Third, one may consider using a different task. This can be an existing (sequential) risk-taking task, like the Columbia Card Task (Figner et al., 2009), which performs better in terms of decomposability than the BART. Alternatively, researchers should consider creating a custom task that exactly suits their research, avoiding methodological flaws that could endanger the soundness of their conclusions. For instance, a task developed by Schmidt et al. (2013) involves decisions under conditions of explicit risk, and does not confound risk with expected value. An important goal to keep in mind when designing such bespoke tasks is to combine strong ecological validity with methodological rigour (Schonberg et al., 2011).
Clearly, none of the solutions proposed can be considered a 'universal' fix that solves all of the BART's problems. Depending on the aims of any given study, certain problems will be more pressing than others, indicating the (type of) solutions most needed. By combining solutions, researchers could work towards a task that can operationalise risk propensity without substantial methodological or interpretational problems. For example, an automatic BART with full feedback and explicit information on the probability distribution provides uncensored decisions made under clear risk that are at times risky enough to be maladaptive. If the resulting data from this adapted BART are then analysed using a model like that by Wallsten et al. (2005) or that by Van Ravenzwaaij et al. (2011), all problems reviewed in the current commentary would be addressed. However, this does not necessarily mean that this combination of solutions constitutes a universal fix after all, as the BART may face more problems than the ones discussed here. In all likelihood, the present review is not exhaustive. Researchers using the BART may know of additional problems, although this is unlikely to show in their work, as journals - and by extension researchers - do not consider 'failure' a popular publishing theme (Ferguson \& Heene, 2012; Song et al., 2009). Therefore, it is important for researchers to not only critically evaluate the instruments they use, but to disclose these evaluations as well, so that any and all methodological shortcomings can be openly discussed and addressed, improving the quality of the measures used.

\section{Conclusion}

The present paper is the first to review the methodological shortcomings of the Balloon Analogue Risk Task, a highly popular risk-taking task in psychology. The main problems identified are the ambiguity between uncertainty and risk, censoring of observations, confounding of risk and expected value, and poor decomposability into adaptive and maladaptive risk-taking. In addition, the paper reviews solutions that mitigate these problems. By presenting this first-time inventory, the paper highlights earlier mentions of problems in the BART as well as proposed solutions. It calls for a critical attitude towards the BART and experimental tasks in general, as their design deserves at least as much attention as the findings they produce. It also sets the agenda for testing and comparing different tasks and task versions, to explore which designs result in the best usability, reliability, and validity, so that risk propensity can be measured in the most accurate way possible. 


\section{Peer Reviews}

Reviewer 1 Michael Young

\section{0) General comments}

- Accept manuscript either as is or with minor modifications.

- First, this paper is the closest I have come to recommending an 'accept as is' for an initial submission during my entire career.

- Yes, there are some additional modifications that could be made (see below and, likely, comments from the other reviewers), but the paper reads excellently as written and nicely integrates a wide body of literature on methodological issues with the BART.

- With that said, let me introduce some minor suggestions that the authors might consider.

1) Risk or Uncertainty?

- I was curious about the statement [at Risk or Uncertainty?, last paragraph] that "one could use a different task in which decisions are either all characterized by uncertainty or risk." Can the BART be modified to create two such versions?

- For example, a version in which people are presented the pop probability before each decision to pump or cash in would only entail risk, whereas a version where the pop probability is unknown and progresses differently on each trial (i.e., each balloon has a different but unknown color in the original Lejuez task) might represent maximal uncertainty although this probability would always need to increase with each pump.

2) Confounding and Decomposability

- [At Confounding and Decomposability, paragraph 2], the authors mention the problem that balloon value increases linearly whereas probability of explosion increases superlinearly, and thus the EV of the balloon changes across trials.

- Yes, that's an issue in the Lejeuz version, but the BART can be easily modified to remove that concern. If the P(explosion) increases linearly, then the EV would be constant.

3) Discussion

- Two issues not considered are that people might pump less because they are fatigued and thus wish to reduce effort or because they want to complete the task sooner for a finite number of balloons.

- E.g., if there are 30 balloons, I could end the task after 30 pumps by pumping once per balloon rather than 10-15 times per balloon.

- Thus, 'risk propensity' might reflect laziness or boredom with the task or a desire to leave early.

\section{Reviewer 2 Sihua Xu}

\section{0) General comments}

- This manuscript summarizes the popularity and qualities of the Balloon Analogue Risk Task, one of the most popular tasks for gauging risk propensity and puts forward and analyzes for shortcomings of the task.

- Authors' analysis and viewpoints are very important and demand, indeed, further investigation. In my opinion, this manuscript has an innovative character. I have some suggestions to improve its quality.

1) Confounding and Decomposability

a. Personally, authors completely regard risk or uncertainty as an isolated concept to discuss the shortcomings of the BART. Some of the authors' opinions are too extreme.

- For instance, the authors believe that the BART confounds risk with the expected value. However, the risk is not completely isolated in reality. Many risk decision-making situations have interaction between risk and expected value. In this respect, the BART has high ecological validity.

b. Besides, authors suggest that the BART requires participants to inflate balloons one pump at a time, it is plausible that they get tired of pumping before reaching the optimum, which may lead to the BART cannot properly differentiate between adaptive and maladaptive risk behavior.

- However, in my opinion, it is precisely this dynamic decision-making process that allows us to discover the cognitive process and neural mechanism of risk dynamics in the BART using cognitive neuroscience methods.

2) The Normative Solution

- Additionally, the authors addressed the key problems of BART by comparing the original version to subsequent modified versions, rather than other risk-taking tasks in detail.

- It seems that the goal of the present study is summarizing problems inherent to the original BART, not targeted at the task itself. It should be noted that these modified versions were used to mitigate only one or two methodological aspects, which may lead to a concern for the validity and reliability of BART, and a trade-off is needed for researchers.

3) Discussion

- Also, the authors assume that participants are not given any information about the explosion probabilities, they first decide under uncertainty, which 
then gradually shifts towards risk as they learn more about the probabilities in the task.

- However, the authors' discussion on the proposition is not enough, because the authors analyze the transition problem in the BART from deciding under uncertainty to deciding under risk, based on the assumption that the decision in the BART includes two stages: uncertain decision and risk decision.

- In my opinion, there is not enough experimental evidence to support this hypothesis. A recent study suggests that this task is not about risk but uncertainty. In the BART, both outcome and probability distribution are unknown, making it an uncertain task (De Groot \& Thurik, 2018).

- A previous study also found that the test-retest reliability of the BART was significantly higher than that of the IGT (Xu et al., 2013), which indicates that it is more difficult for participants to learn and master the probability information of the BART during the decision-making process.

4) Conclusion

- Finally, in the conclusion section, the authors put forward some methods and advice to overcome the shortcomings, but in my opinion, it is still not detailed and meaningful enough and needs further improvement. 